\title{
Ninth order block hybrid collocation method for second order ordinary differential equations
}

\begin{abstract}
A ninth order block hybrid collocation method is proposed for solving general second order ordinary differential equations directly. The derivation involves interpolation and collocation of basic polynomial that generates the main and additional methods. These methods are applied simultaneously to provide approximate solutions at five main points and three offstep points. The stability properties of the block method are discussed. Some illustrative examples are given to demonstrate the efficiency of the method.
\end{abstract}

Keyword: Ninth order block hybrid collocation method; Second order ordinary differential equations; Block method 